\title{
Ultrafast Laser Induced Molecular Alignment and Deformation: Experimental Evidence from Neutral Molecules and from Fragment Ions ${ }^{\dagger}$
}

\author{
Matthew Comstock, Vahan Senekerimyan, and Marcos Dantus* \\ Department of Chemistry and Center for Fundamental Materials Research, Michigan State University, \\ East Lansing, Michigan 48824
}

Received: December 23, 2002; In Final Form: May 30, 2003

\begin{abstract}
The alignment and molecular structure deformations induced by intense off-resonance excitation with ultrafast laser pulses are examined using femtosecond transient grating spectroscopy and by angle resolved multiphoton ionization in a molecular beam. The goal of this study is to correlate evidence obtained from the angular dependence of multiphoton ionization and from rotational recurrences observed in neutral molecules regarding alignment and molecular structure deformation. Structural parameters are determined by analysis of the fieldfree rotational recurrences, obtained by transient grating measurements, or by analysis of the anisotropy in the detected fragment ions, in the molecular beam experiments. Experimental data were obtained for $\mathrm{CS}_{2}$, $\mathrm{CO}_{2}$, acetylene, and benzene, for pulse intensities ranging from $10^{11}$ to $10^{14} \mathrm{~W} / \mathrm{cm}^{2}$. The experimental results are consistent with molecular alignment resulting from a "kick" induced by the ultrafast off-resonance field. The results also provide evidence of molecular structure deformations. Results from transient grating experiments indicate that the electric field can induce alignment and bending in polyatomic molecules and that these effects can take place in the absence of ionization.
\end{abstract}

\section{Introduction}

High-intensity off-resonance laser fields from ultrafast laser pulses can cause very large electric field gradients. Molecules can experience a large torque along the polarization vector of the field due to their anisotropic polarizability. With long laser pulses the torque is enough to cause adiabatic alignment; ${ }^{1,2}$ however, with ultrafast pulses the torque provides an "instantaneous" kick toward alignment. ${ }^{3}$ Pulses with peak intensities of $10^{13} \mathrm{~W} / \mathrm{cm}^{2}$ generate electric fields of $1 \mathrm{~V} / \AA$, causing significant changes in the electronic energy levels of the molecule. When the electronic states have different geometry, the fast strong field excitation process leads to molecular structure deformation. In this report we study alignment and deformation for a number of molecular species using the time resolved transient grating (TG) method. For $\mathrm{CS}_{2}$, we obtain angle resolved ion yields following Coulomb explosion in the molecular beam and find evidence for the molecular deformation.

This study bridges these two types of measurements and provides experimental evidence regarding alignment and structural deformation. The results presented here shed new light on the understanding of the behavior of molecules in the presence of intense and ultrashort laser fields. ${ }^{4}$

In Figure 1, we illustrate the dependence of the electric field trap depth measured in Kelvin for molecules with relatively large anisotropic polarizability (for example, $\mathrm{CS}_{2}$ ). Laser intensities of $10^{11} \mathrm{~W} / \mathrm{cm}^{2}$ are enough to align cold (10 K or less) molecules, for example, from molecular beams..$^{5}$ Also shown in Figure 1 is the electric field strength as a function of the peak intensity of the laser. The electric field is calculated using $E=\left(2 I Z_{0}\right)^{1 / 2}$, where $Z_{0}$ is the resistivity of a vacuum $(376.7 \Omega)$ and $I$ is the

\footnotetext{
† Part of the special issue "A. C. Albrecht Memorial Issue".

* To whom correspondence should be addressed. E-mail: dantus@ msu.edu.
}

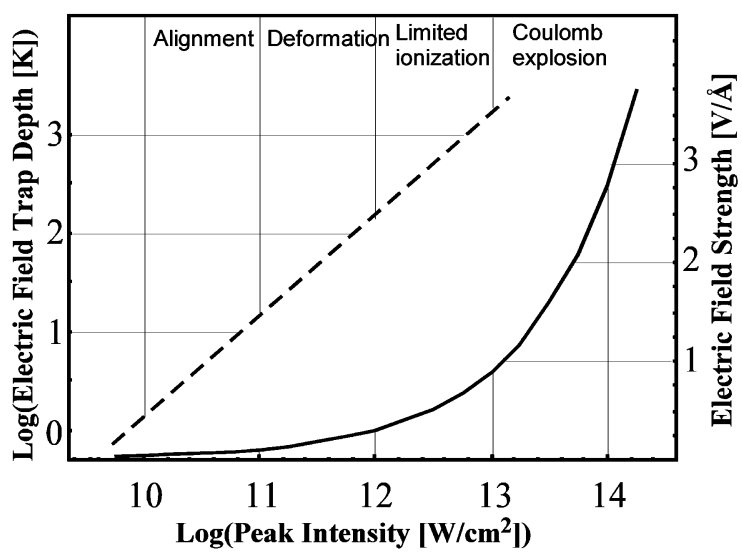

Figure 1. Plot of the induced rotational trap depth on a highly polarizable molecule like $\mathrm{CS}_{2}$ (dotted line, left axis) and the electric field strength (solid line, right axis) as a function of peak laser intensity. The predominantly observed molecular processes that occur at any given intensity are indicated at the top.

peak intensity of the laser pulse. For laser intensities of $10^{12}$ $\mathrm{W} / \mathrm{cm}^{2}$, the electric field can exert sufficient force on a molecule to cause alignment. Higher peak intensity fields, $\sim 10^{13} \mathrm{~W} / \mathrm{cm}^{2}$, produce an electric field strength that is comparable to that experienced by valence electrons in molecules $(\sim 1 \mathrm{~V} / \AA)$, and structural deformation takes place. At these electric field strengths, ionization takes place, and at higher laser intensities, multiple ionization and Coulomb explosion are observed.

The observation of molecular alignment stemming from highintensity nonresonant laser excitation has been theoretically explored by Friedrich and Hershbach. ${ }^{1,6}$ The intense laser field causes an induced dipole in the molecule, which suppresses the rotational motion and leads to aligned pendular states. ${ }^{1,6}$ Friedrich and Herschbach developed the laser induced alignment theory based on earlier observations of dissociative multiphoton 
ionization experiments on $\mathrm{CO}$ and $\mathrm{I}_{2}$ with intense infrared lasers conducted by Normand et al. ${ }^{7}$ and Dietrich et al., ${ }^{8}$ respectively. Demonstrations of the laser induced alignment technique have been conducted by Kim and Felker on large nonpolar molecules $^{2,9}$ and by Stapelfeldt and co-workers on smaller molecules. ${ }^{10,11}$ Posthumus et al. have examined the multiphoton dissociative ionization of $\mathrm{H}_{2}, \mathrm{~N}_{2}$, and $\mathrm{I}_{2}$ with 50 fs pulses and found that only $\mathrm{H}_{2}$ and $\mathrm{N}_{2}$ showed alignment characteristics. ${ }^{12}$ Corkum and co-workers have combined intense off-resonance chirped circularly polarized fields to induce rotational acceleration and thus constructed a molecular centrifuge. ${ }^{13}$ Alignment with resonant intense laser fields has been examined theoretically by Seideman. When on-resonance fields are used, the intensity does not have to be as strong as that in off-resonance experiments to generate alignment. ${ }^{14}$ She noted that as the mass of the molecule increased (and thus the rotational period increased), longer pulse duration was required to align the molecules. The study by Brown et al., on the resonant excitation of $\mathrm{I}_{2}$ using 50 fs pulses, confirmed the propensity for multiphoton excitation rather than alignment for a relatively heavy molecule. ${ }^{15}$ Nonresonant excitation is advantageous, especially with larger molecules, because there is less propensity for ionization. ${ }^{14}$ Ortigoso et al. calculated the conditions (laser pulse and rotational constant of the molecule) for which recurrences of alignment can be expected with nonresonant short pulses. ${ }^{16}$

One of the most widely used methods for studying the behavior of molecules under intense laser fields is the Coulomb explosion (CE) method. In the CE method, the ultrafast intense field causes multiple ionization of the parent molecule. Mapping the distribution of the fragment ions gives information about the molecular structure prior to explosion. ${ }^{17-33}$ While very powerful, it is very difficult to determine a sequence of events leading to the observed fragment distribution using the $\mathrm{CE}$ method. For example, it is important to determine if structural deformation takes place in the neutral molecules or it arises after ionization. Similarly, it is important to determine the laser intensity that causes alignment and deformation, an aspect that is difficult to determine by the CE method. This specific question is addressed in this study.

Research from our group has taken advantage of the experimental measurement of rotational recurrences following the formation of an off-resonance polarization transient grating, a method introduced by Heritage et al. ${ }^{34}$ With femtosecond pulses one is able to make much more accurate determinations of rotational constants and hence better determination of molecular structure. ${ }^{35-37}$ Soon after, we recognized that the transient grating method would allow us to measure alignment and molecular deformation induced by the strong off-resonance field provided the third pulse (the probe) was kept at a low and constant intensity while the first two pulses, which coincide in time and form the transient grating, provide the strong field. This setup was used to make our first observations of alignment and structural deformation following intense off-resonance excitation. $^{38-40}$

The presentation is organized as follows. We present briefly the laser systems and experimental setups used for our measurements. In the Results section we present results obtained by time resolved transient grating measurements. We then present results obtained from strong field multiphoton excitation of $\mathrm{CS}_{2}$ and analyze the angular distribution of the fragment ions. In the Discussion we correlate the different measurements and analyze them in terms of alignment and structural deformation. Because these two experimental methods probe different species (ion fragments in Coulomb explosion and neutrals in transient grating), it is important to determine if there is good agreement between the two and by comparison learn about the time scales of molecular alignment and structural deformation, as compared to ionization and Coulomb explosion. Finally, we draw conclusions about the effect of strong laser fields on molecules and about the time scale for both structural deformation and ionization in these experiments.

\section{Experimental Section}

The data presented come from two different laser systems. Most of the data were acquired with a home-built colliding pulse mode-locked dye laser (CPM), pumped by a CW Nd:YLF laser. The output from the CPM is centered at $620 \mathrm{~nm}$ and is amplified using a four-stage dye amplifier pumped by a $30 \mathrm{~Hz} \mathrm{Nd}$ :YAG laser. A double-pass prism pair recompresses the pulses after amplification to produce $60 \mathrm{fs}$ pulses. For the CE experiments we double-passed the fourth stage to obtain greater energies per pulse but longer pulses, $\sim 80 \mathrm{fs}$. The pulse energy used for each experiment is indicated in the text. The transient grating experiments were obtained using the forward box geometry. The first two high-intensity laser pulses coincided in time and were followed by a weak probe pulse delayed by a computer controlled actuator. The three pulses were horizontally polarized. The signal registered corresponds to the diffraction of the probe laser from the transient grating formed by the two intense pulses. ${ }^{37}$ The degree of molecular alignment and deformation are measured from changes in the rotational recurrence intensity and shape observed after field-free evolution of the rotational wave packets. The data for $\mathrm{CO}_{2}$ were obtained using a regeneratively amplified titanium sapphire laser producing $1 \mathrm{~mJ}$, $50 \mathrm{fs}$ pulses centered at $800 \mathrm{~nm}$.

For TG experiments (see Figure 2, top) the intensity of the first two laser pulses was varied between $10^{10}$ and $10^{13} \mathrm{~W} / \mathrm{cm}^{2}$ using a set of matched neutral density filters, all with the same substrate thickness. The intensity of the third pulse, the probe, was maintained at a fixed $10^{10} \mathrm{~W} / \mathrm{cm}^{2}$. The signal was collected background-free at the phase matching geometry. The samples were introduced in a $10 \mathrm{~cm}$ long cylindrical quartz cell. In some cases, for example in $\mathrm{CS}_{2}$, a coldfinger in the cell was kept in ice to reduce the sample density. Sample densities were 400 Torr for $\mathrm{C}_{2} \mathrm{H}_{2}$ and $\mathrm{CO}_{2}, 100$ Torr for $\mathrm{CS}_{2}$, and 150 Torr for $\mathrm{C}_{6} \mathrm{H}_{6}$.

Ionization data were obtained using a molecular beam apparatus (see Figure 2, bottom). The samples were introduced using $\mathrm{He}$ as a carrier gas, which entrained some of the vapor pressure of the sample. The sample and carrier gas (He, 1700 Torr) expanded through the $1 \mathrm{~mm}$ diameter aperture in the pulsed valve. A $2 \mathrm{~mm}$ diameter skimmer produced the molecular beam, which proceeded to the ionization chamber. The pulses $0.25 \mathrm{~mJ}$ in energy were focused onto the molecular beam by an $f=150 \mathrm{~mm}$ lens. The diffraction size of the beam in the focal plane is estimated at $20 \mu \mathrm{m}$, and the experimentally determined beam size is $26 \mu \mathrm{m}$. At the focus, the peak power on the molecular beam then is $4.6 \times 10^{14} \mathrm{~W} / \mathrm{cm}^{2}$. The voltages on the extractor and repeller plates were kept at 1800 and $2500 \mathrm{~V}$, respectively, with a distance between them being equal to $1.5 \mathrm{~cm}$. The pressure in the ionization chamber was $\sim 8 \times$ $10^{-8}$ Torr when the valve was closed and $\sim 7 \times 10^{-7}$ Torr when the valve was operated at $30 \mathrm{~Hz}$. The ions were allowed to travel in a drift-free zone of $50 \mathrm{~cm}$ before being detected by a microchannel plate. Twenty time-of-flight mass spectra (TOFMS) were averaged before they were analyzed. The polarization dependence of the ion intensities was obtained taking advantage of a zeroth-order half-wave plate, which was rotated manually 

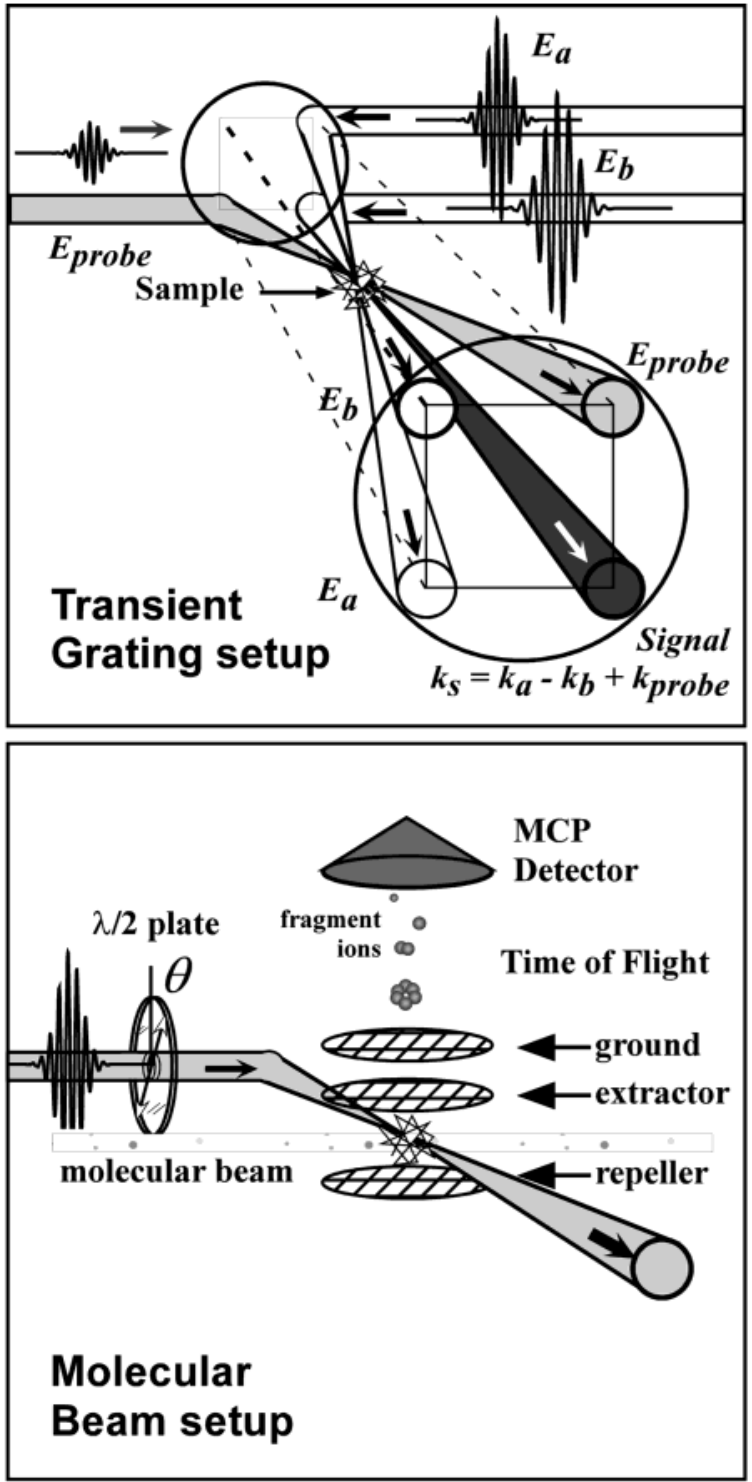

Figure 2. Schematic drawing of the experimental setup for time resolved transient grating (top) and molecular beam (bottom) experiments. The transient grating signal was detected at the phase matching angle as a function of the time delay between the first two strong field pulses and the weak probe. Ion detection, in the molecular beam, was recorded as a function of the angle that the polarization vector of the laser pulse makes with the time-of-flight axis.

from 0 to $180^{\circ}$ in $15^{\circ}$ increments with respect to the time-offlight axis. A calcite polarizer was used to ensure very high polarization purity (greater than 1:10000) of the beam.

\section{Results}

A. Evidence of Alignment and Structural Deformation from Transient Grating Measurements. Three-pulse transient grating experiments were carried out to explore the occurrence of alignment and structural deformation. The full rotational recurrence of $\mathrm{CS}_{2}$ is expected at $76.5 \mathrm{ps}^{34,37}$ When the laser pulses are weak, this feature can be observed and accurate rotational constants can be determined..$^{37,40-44}$ In Figure 3a, we show experimental data for the case when all laser pulses are weak. The simulation of the data with no adjustable parameters fits well the experimental results (see Figure 3a). When the laser intensity of the first two pulses is increased by an order of magnitude, the full rotational recurrence observed increases in intensity. The data obtained at this intensity can still be fitted


Figure 3. First rotational recurrence of $\mathrm{CS}_{2}$ at three different laser intensities. Probe intensity was held constant throughout at $2 \times 10^{10}$ $\mathrm{W} / \mathrm{cm}^{2}$. (a) Low-intensity data (circles) and simulation (solid line) based on unperturbed molecules. (b) Data (circles) under higher intensity indicating laser induced alignment. The simulation (solid line) assumes an unperturbed molecular geometry. (c) High-intensity data (circles) showing a change in the observed rotational revival, evidence for structural deformation of the $\mathrm{CS}_{2}$ molecule. The solid line in part $\mathrm{c}$ is a simulation based on $35 \%$ of the sample having a bond angle of $160^{\circ}$.

by a room-temperature distribution of linear $\mathrm{CS}_{2}$ molecules (see Figure $3 b$ ). Under these conditions, signal intensity can only increase if there is a larger anisotropic polarization of the sample. This higher polarization results from a greater number of molecules being pulled into alignment by the electric field. Previous work describing the time scales needed for alignment has considered the very low temperature available in molecular beams. ${ }^{5,45}$ At the higher temperatures here, the time scale of molecular rotation is much closer to the pulse duration of the laser. The rms rotational time of $\mathrm{CS}_{2}$ at room temperature is $1.8 \mathrm{ps}\left(J_{\mathrm{rms}}=62\right)$. This means that, during the $60 \mathrm{fs}$ laser pulse, a molecule with the rms rotational energy can rotate $15^{\circ}$.

Simulations of TG data presented here follow the quantum mechanical expression for the time dependent susceptibility, $35-37,46$

$$
\begin{array}{r}
P(t) \propto N \beta \mathrm{T} \Sigma_{v} \Sigma_{J} q_{v} \rho_{J}\left\{\cos \left(2 \pi c\left(F_{J+2}-F_{J}\right) t\right)+\right. \\
\left.\cos \left(2 \pi c\left(F_{J}-F_{J-2}\right) t\right)\right\}
\end{array}
$$

where $F_{J}=B_{v} J(J+1)-D J^{2}(J+1)^{2}$ for the molecule, ${ }^{47} B_{\mathrm{v}}$ is the vibrationally dependent rotational constant, $D$ is the 
centrifugal distortion constant, $\beta$ is the anisotropic polarizability, $T$ is the temperature, $N$ is the number density, $\rho_{J}$ is the normalized rotational distribution including spin statistics $\left(g_{J}\right)$ for all natural isotopes, ${ }^{48} \rho_{J}=g_{J}(2 J+1) \exp \left(-2 \pi c h F_{J} / k T\right) /$ $\left\{\Sigma_{J} g_{j}(2 J+1) \exp \left(-2 \pi c h F_{J} / k T\right)\right\}$, and $q_{\mathrm{v}}$ is the normalized thermal vibrational distribution for all ground-state vibrational modes. Due to the off-resonance nature of the process, $\Delta J=$ $0, \pm 2$, rotational recurrences are seen every $\tau_{\text {rec }}=1 /(4 B c)$. The homodyned TG signal is given by $\left|(k T)^{-1} \mathrm{~d} P / \mathrm{d} t\right|^{2}$ while the heterodyned TG signal is given by $-(k T)^{-1} \mathrm{~d} P / \mathrm{d} t .^{35-37,46}$ For samples containing molecules with different rotational constants, the homodyned signal is given by $\left|(k T)^{-1}\left(\mathrm{~d} P_{1} / \mathrm{d} t+\mathrm{d} P_{2} / \mathrm{d} t\right)\right|^{2}$, leading to interference terms between the two populations. ${ }^{40}$ We have used eq 1 to simulate all the transient grating data. For high-intensity excitation, some deviations are expected in the rotational population distribution caused by Rabi cycling (multiple Raman transitions). Because our experiments are carried out at room temperature, with a broad initial rotational distribution, a very large number of Rabi cycles $(>100)$ would be needed to observe a change in the recurrence structure.

When the intensity of the first two pulses is increased above $10^{12} \mathrm{~W} / \mathrm{cm}^{2}$, the full rotational recurrence increases in intensity and changes shape (see Figure 3c). Most markedly, the first feature increases in intensity by a factor of 2 . The features observed at long time delays, $>77.5 \mathrm{ps}$, increase in intensity, and new oscillations are observed. Attempts to explain the observed changes in the rotational recurrence structure by changes in the rotational population caused by Rabi cycling in the strong field in the manner of Rosca-Pruna and Vrakking ${ }^{49,50}$ have been shown to be inadequate to explain our experimental results. ${ }^{40,51} \mathrm{~A}$ broadened rotational population leads to narrowing of the rotational recurrence and to a lower intensity of the first feature; our data show the opposite behavior. ${ }^{39,40,51}$ Similarly, bond softening has been considered and found to be inconsistent with our data. ${ }^{40,51}$ Simulation of the data for high-intensity excitation shown in Figure $3 \mathrm{c}$ is compatible with the presence of bent molecules. A static bond angle of $160^{\circ}(B=0.110536$ $\mathrm{cm}^{-1}, C=0.109995 \mathrm{~cm}^{-1}$ ) was used in our simulation to reproduce the experimental data. The quality of the fit together with evidence from the $\mathrm{CE}$ experiments presented below strongly supports field induced bending of the $\mathrm{CS}_{2}$ molecules.

B. Evidence of Structural Deformation for Coulomb Explosion. To corroborate the observed molecular structure changes, we studied the effects induced by the strong field in Coulomb explosion experiments. In Figure 4a, we show the TOF-MS resulting from excitation with pulses with $4.6 \times 10^{14}$ $\mathrm{W} / \mathrm{cm}^{2}$ peak intensity. In addition to the parent ions $\left(\mathrm{CS}_{2}{ }^{+}\right)$, $\left(\mathrm{CS}_{2}{ }^{2+}\right)$ multiple fragments are observed. We distinguish the singly ionized fragments $\left(\mathrm{C}^{+}\right.$and $\left.\mathrm{S}^{+}\right)$from the doubly ionized fragments $\left(\mathrm{C}^{2+}\right.$ and $\left.\mathrm{S}^{2+}\right)$. In Figure $4 \mathrm{~b}$, we show the ionization yield of the parent ion as a function of the polarization vector of the excitation beam. Notice that the yield is isotropic, as expected, because of the isotropic distribution of $\mathrm{CS}_{2}$ molecules in the molecular beam. The data in Figure $4 \mathrm{~b}$ serve also to confirm that there is no systematic bias in the probability of ion detection as a function of the polarization vector of the excitation field with respect to the time-of-flight axis. A similar isotropic distribution was observed for the $\mathrm{CS}_{2}{ }^{2+}$ ion.

The angle dependent ionization yield for $\mathrm{S}^{+}$and for $\mathrm{C}^{+}$was measured, and the results are presented in Figure 5 in Cartesian and polar coordinates. The experimental data obtained for three separate runs are depicted as circles, squares, and triangles. The lines are best fitted by cosine or sine functions to the fourth
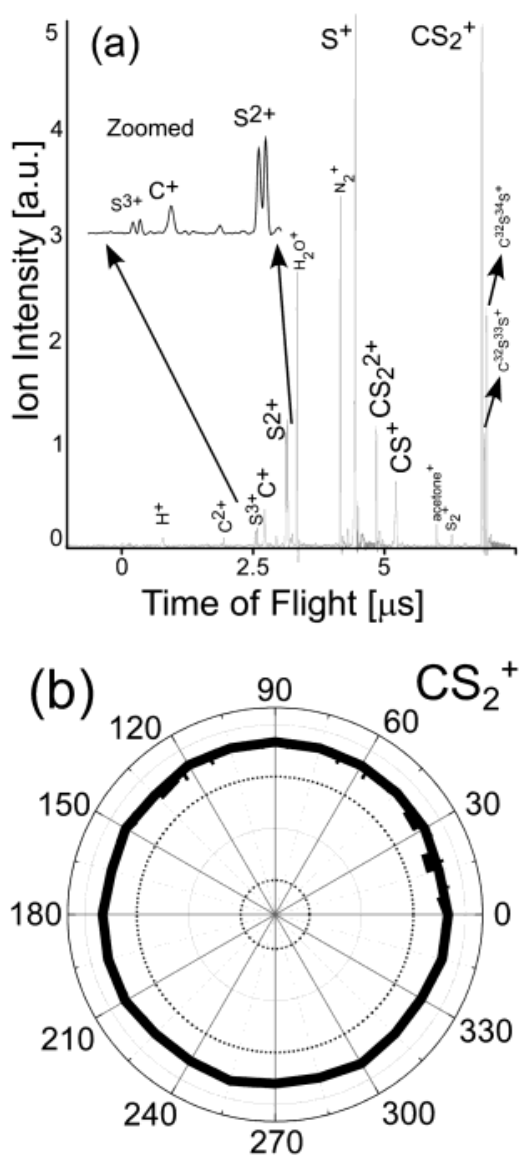

Figure 4. Time-of-flight mass spectrum obtained for $\mathrm{CS}_{2}$ molecules (a) when the laser polarization vector is aligned with the TOF axis. The zoomed inset shows splitting of the sulfur fragment ion peaks caused by their Coulomb explosion toward and away from the detector. (b) Angle dependent distribution of $\mathrm{CS}_{2}{ }^{+}$ions, showing an isotropic distribution.

power. Notice that the yield for $\mathrm{S}^{+}$follows a cosinusoidal distribution, with maxima at zero and $180^{\circ}$ (see Figure 5a). The yield for $\mathrm{C}^{+}$ions follows a sinusoidal distribution with maxima at 90 and $270^{\circ}$ (see Figure 5b).

The angle dependent ionization yield for $\mathrm{S}^{2+}$ and for $\mathrm{C}^{2+}$ was measured, and the results are presented in Figure 6 in Cartesian and polar coordinates. The experimental data obtained for three separate runs are depicted as circles, squares, and triangles. The lines are best fitted by cosine or sine functions to the fourth power. Notice that the yield for $\mathrm{S}^{2+}$ follows a cosinusoidal distribution, with maxima at zero and $180^{\circ}$ degrees (see Figure 6a). The yield for $\mathrm{C}^{2+}$ ions follows a sinusoidal distribution with maxima at 90 and $270^{\circ}$ (see Figure 6b). Notice that the results for the doubly ionized fragments show greater sensitivity toward the angle of the polarization vector of the excitation pulse compared to the case of the singly ionized fragments in Figure 5. This indicates the doubly charged particles are ejected from a multiply charged parent with more significant bending than that of the singly charged ions.

The ionization data shown in Figures 5 and 6 are consistent with the angular distribution of the fragment ions obtained with a titanium sapphire laser centered at $800 \mathrm{~nm} .{ }^{26,27}$ Similarly, we are able to interpret our data in terms of a molecular structure deformation that is consistent with bending. The angular dependence observed in the molecular beam is consistent with the molecular structure deformations measured by the TG method on the neutral molecules. 

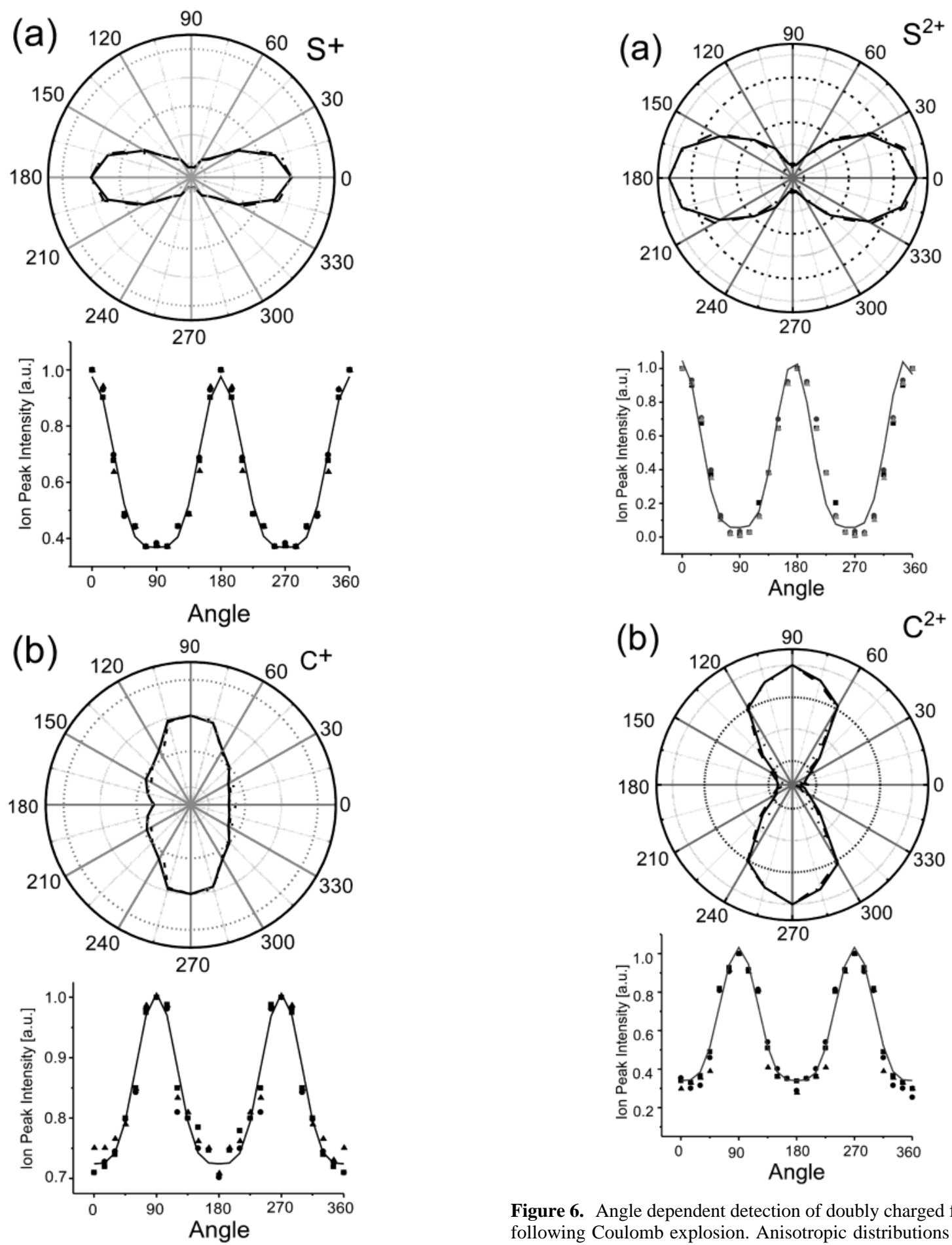

Figure 6. Angle dependent detection of doubly charged fragment ions following Coulomb explosion. Anisotropic distributions are observed for $\mathrm{C}^{2+}$ (a) and $\mathrm{S}^{2+}$ (b) ions in polar and Cartesian plots. Data for three independent experiments (squares, circles, triangles) are accompanied


shows a maximum ion yield at $0^{\circ}$ and $180^{\circ}$, while $\mathrm{C}^{2+}$ shows a maximum at $90^{\circ}$ and $270^{\circ}$. independent experiments (squares, circles, triangles) are accompanied

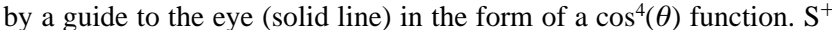
shows a maximum ion yield at $0^{\circ}$ and $180^{\circ}$, while $\mathrm{C}^{+}$shows a maximum at $90^{\circ}$ and $270^{\circ}$. The anisotropy for the $\mathrm{C}^{+}$ions is less pronounced.

C. Alignment and Deformation of Polyatomic Molecules. In Figure 7 we turn our attention to the behavior of $\mathrm{CO}_{2}$ molecules under strong field excitation. These experiments were carried out with a titanium sapphire laser centered at $800 \mathrm{~nm}$. For low intensities (Figure 7a) the rotational recurrence can be fit by a simulation of $\mathrm{CO}_{2}$ molecules at room temperature with overall signal intensity as the only adjustable parameter. As the

laser intensity is increased (Figure $7 b-d$ ), the rotational revival gains in intensity and changes overall shape. Increases in intensity can be directly related to laser induced alignment, while changes in the shape of the revival are directly linked to changes in the molecular structure. On the basis of our simulation, the angle of the deformed $\mathrm{CO}_{2}$ molecules is $161 \pm 0.5^{\circ}$. For stronger fields (Figure $7 \mathrm{e}-\mathrm{g}$ ) a background signal is observed and the shape of the revival changes substantially. The background is due to ionization and plasma formation, and it is modeled in our simulations by an emission with an exponential decay of 

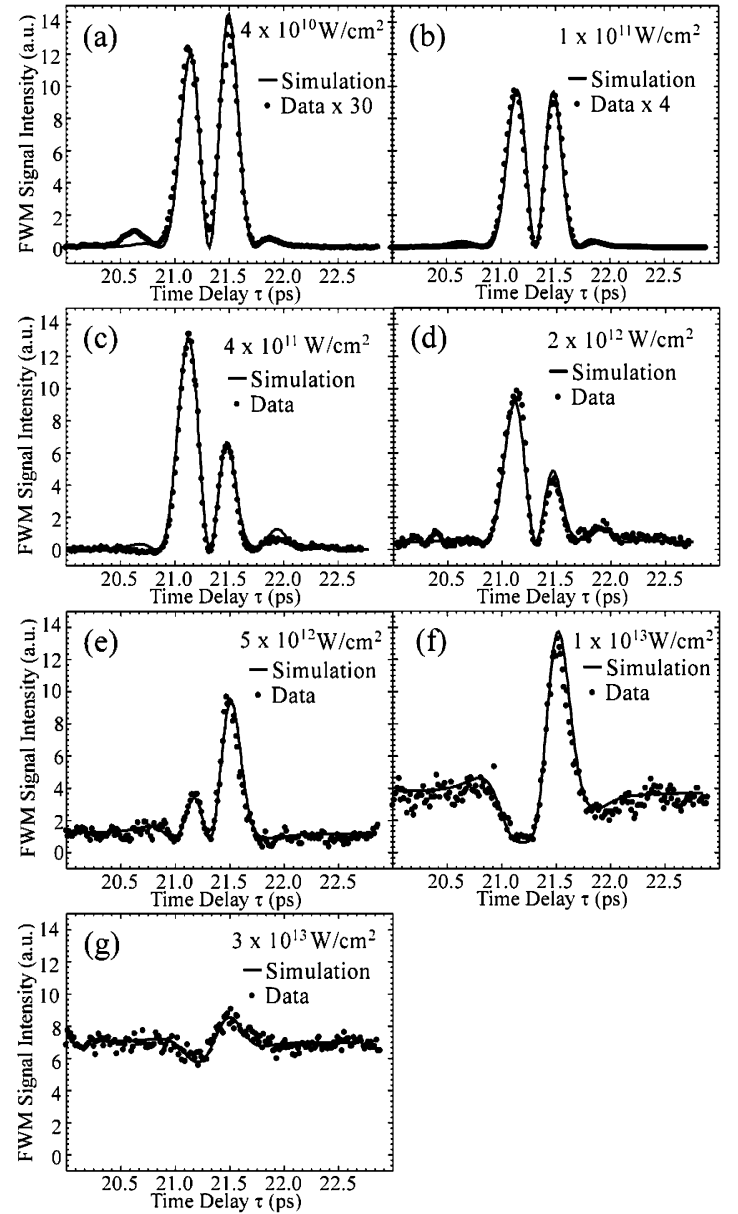

Figure 7. Time resolved TG experimental data centered on the first rotational recurrence of $\mathrm{CO}_{2}$, with the probe intensity held constant at $2 \times 10^{10} \mathrm{~W} / \mathrm{cm}^{2}$. At low intensities (a and b), the data (circles) match well the simulation (solid line) based on an unperturbed linear molecule. Near $10^{12} \mathrm{~W} / \mathrm{cm}^{2}$ ( $\mathrm{c}$ and d), the data show evidence of bending. The solid lines in parts $\mathrm{c}$ and $\mathrm{d}$ are simulations assuming $35 \%$ of the sample has a bond angle of $160^{\circ}$. At higher intensity (e, f, and g), the signal appears above a constant background signal. The simulation assumes that molecules in the intense field have been destroyed and the signal arises from unperturbed linear molecules heterodyned by a constant background.

$1 \mathrm{~ns}$, added coherently to the rotational recurrence signal. The presence of a coherent background signal has been observed before in high-intensity four-wave mixing experiments. ${ }^{43,52,53}$ The rotational recurrences are deformed by interference between the plasma emission and the TG signal. This aspect will be discussed below.

In Figure 8 we analyze the effect of strong field excitation at $620 \mathrm{~nm}$ on acetylene. As the laser intensity is increased, the intensity of the rotational revivals increases. Again, this increase is directly linked to laser induced alignment. For the higher intensity data (Figure 8c), the intensity has increased by a factor of almost 480 and some deformation is observed. Interestingly, the main deformation is a broadening of the first feature of the rotational revival.

Finally, we have explored the structural deformation of benzene as a function of $620 \mathrm{~nm}$ laser intensity (see Figure 9). The data obtained for low intensities (Figure 9a) can be fit with the spectroscopic parameters of room-temperature benzene. For higher intensity excitation, we do not observe a large change in the amplitude of the features, indicating that the alignment observed is not as substantial as that observed for the linear molecules. Already, for intermediate intensities (Figure 9b) the
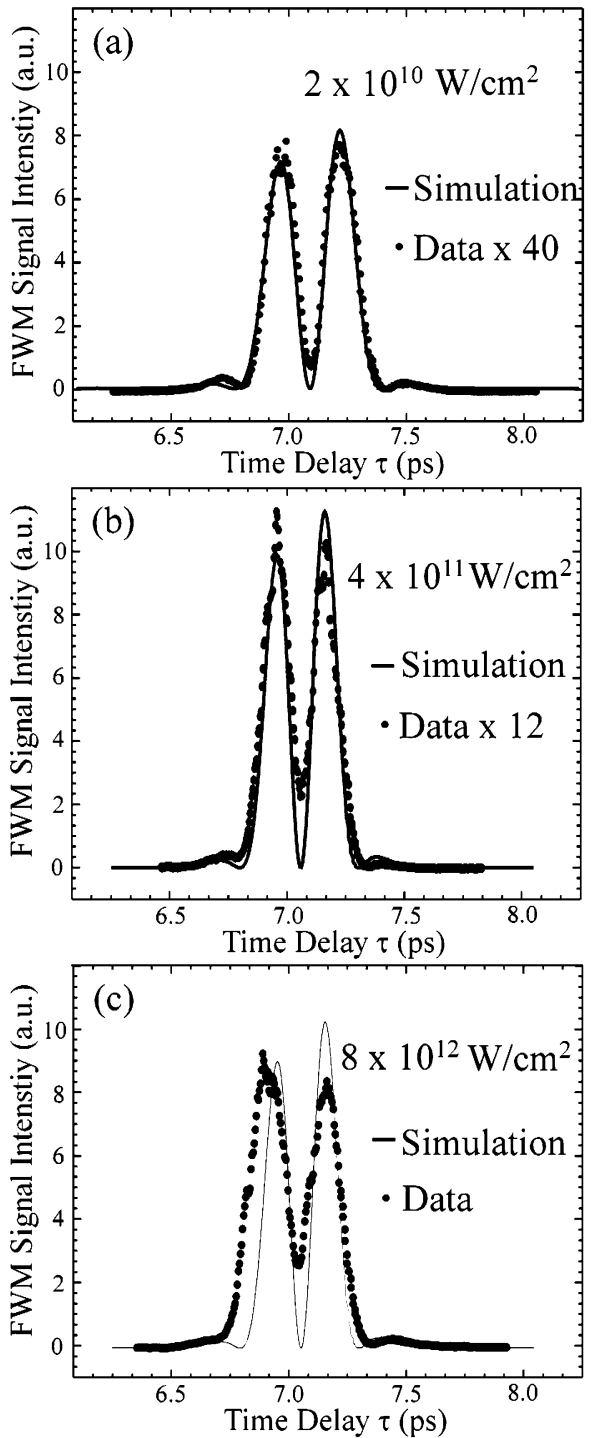

Figure 8. Time resolved TG experimental data centered on the first rotational recurrence of acetylene, with the probe intensity held constant at $2 \times 10^{10} \mathrm{~W} / \mathrm{cm}^{2}$. At low intensity (a), the data (circles) match well the simulation (solid line) based on unperturbed molecules. As the laser intensity is increased (b and c), the first peak of the recurrence broadens, beginning to overlap with the second peak. The factor of 40 in intensity from parts a to $\mathrm{c}$ can be explained by an enhanced alignment of the acetylene molecules in the laser field.

first feature in the rotational revival is broadened toward early times; a similar deformation was observed for acetylene. For higher excitation energies, the signal level increases by a factor of 2 and there is substantial molecular deformation that cannot be explained by a coherent background signal.

\section{Discussion}

There are usually two views of off-resonance laser induced alignment. The first one involves adiabatic alignment induced by a slowly increasing electric field (long laser pulse) that creates a directional force that traps the molecules in pendular states. ${ }^{6}$ As the electric field strength is reduced to zero, the rotational distribution in the sample is restored unaffected; hence, the adiabatic terminology is used. This picture is consistent with experiments involving a nanosecond laser pulse as the source of the strong field. The second view involves an instantaneous "kick" toward alignment, a view that is consistent with the interaction of an ultrashort pulse with supersonically cooled 

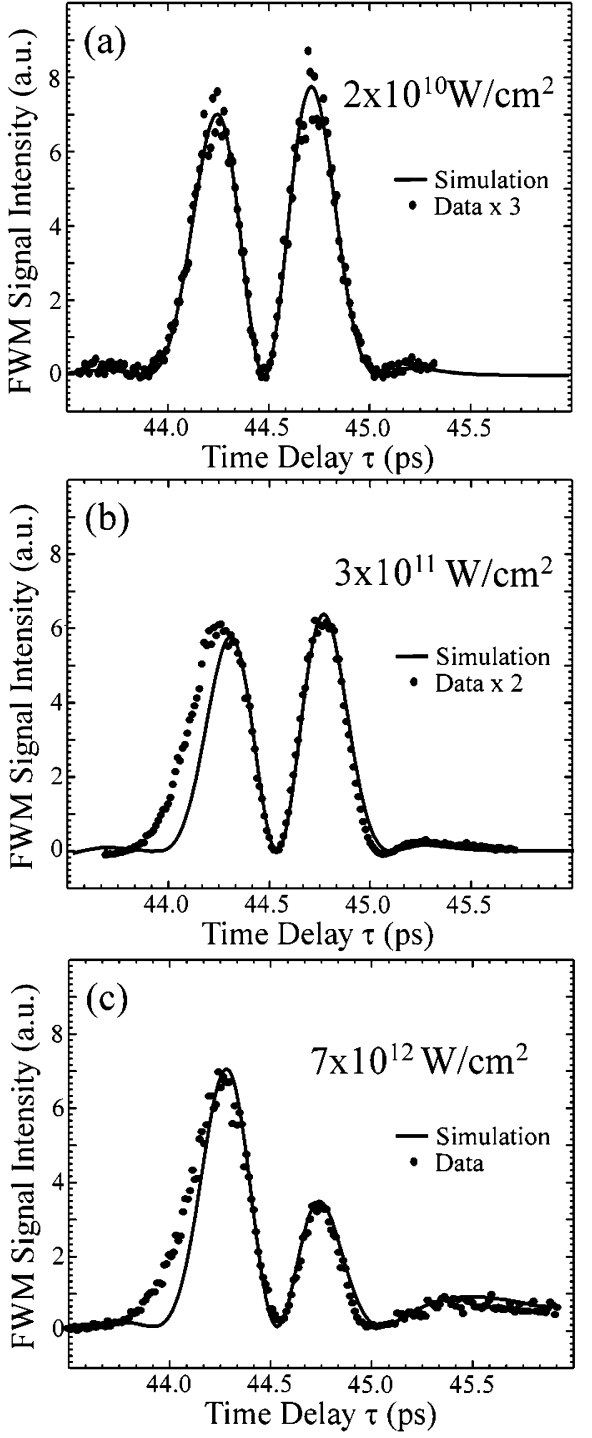

Figure 9. Time resolved TG experimental data centered on the first rotational recurrence of benzene, with the probe intensity held constant at $2 \times 10^{10} \mathrm{~W} / \mathrm{cm}^{2}$. When the grating intensity is low (a), the data (circles) are in good agreement with the simulation (solid line) assuming unperturbed molecules. At $3 \times 10^{11} \mathrm{~W} / \mathrm{cm}^{2}(\mathrm{~b})$, the first peak of the recurrence broadens, and by $7 \times 10^{12} \mathrm{~W} / \mathrm{cm}^{2}$, there is evidence for significant deformation of the benzene molecule. The simulation in part c assumes a symmetric top with a $c_{3}$ symmetry axis (ring puckering mode).

molecules. ${ }^{45}$ The experiments presented using the TG method at room temperature are in an intermediate regime. Molecules with low angular momentum feel a kick toward alignment, essentially not moving while the field is on. Molecules with high angular momentum are able to rotate on the time scale of the laser pulse, and therefore, their alignment is more consistent with the adiabatic picture. The data shown in the Results section are consistent with alignment induced by strong off-resonance electromagnetic fields. As shown in Figure 1, the trap depth as a function of peak intensity reaches hundreds of $\mathrm{K}$ for lasers with peak intensity $\sim 10^{12} \mathrm{~W} / \mathrm{cm}^{2}$ and the resulting alignment of the room-temperature molecules becomes possible.

In addition to alignment, the data presented here are consistent with large molecular structure deformations. In the case of linear molecules, bending can be understood in terms of electronic state mixing. For $\mathrm{CS}_{2}$ and $\mathrm{CO}_{2}$, singlet and triplet excited states are bent. In Figure 10 we show a schematic representation, from a "dressed state" point of view, ${ }^{54,55}$ leading to molecular

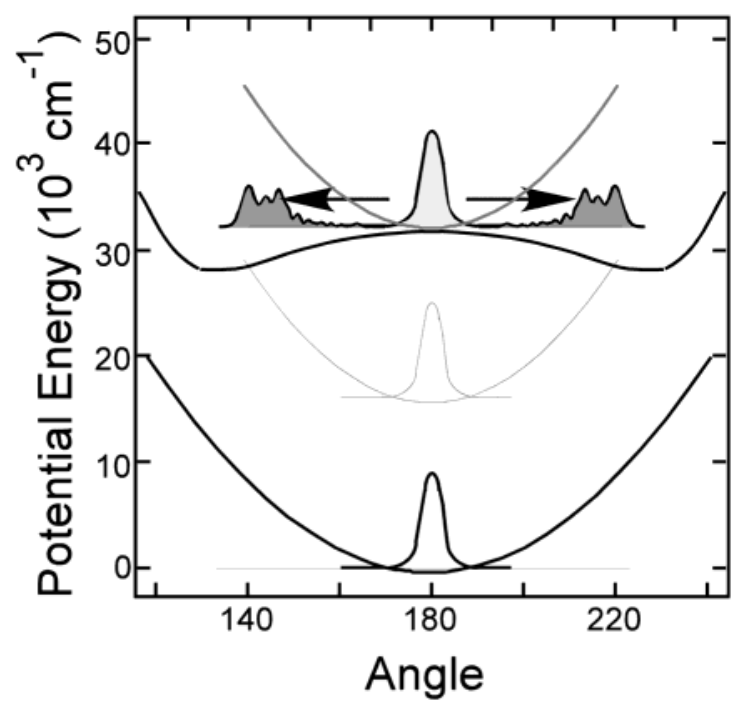

Figure 10. Schematic representation of the laser induced molecular deformation process of $\mathrm{CS}_{2}$ molecules based on a dressed state picture. The laser dresses the ground state and brings it close to the excited states having a bent geometry. The wave packet has a short time to evolve in the dressed state potential during the laser pulse. The system returns to the ground state with substantial bending vibrational energy, leading to a deviation from linear geometry and producing a higher rotational constant.

deformation. Potential energy curves in the absence of external fields are available in the literature. ${ }^{56-58}$ In the figure we show the electronic ground state ${ }^{59}$ as dressed by two $620 \mathrm{~nm}$ photons and overlapping the bent ${ }^{1} \mathrm{~B}_{2}$ electronic state. The ground state wave packet, originally at the equilibrium geometry, evolves within the pulse duration, $\sim 60 \mathrm{fs}$, away from linear geometry. Once the field is turned off, the molecules are left with high amplitude vibrational excitation. Therefore, the rotational recurrence indicates a deviation from the linear geometry.

One of the advantages of the TG method to assess molecular deformation following strong field excitation is that the molecular geometry can be measured long after the field has been turned off. The molecules rotate field-free, and their rotational revival reveals the average bent angle (the deviation from linear geometry). In the case of $\mathrm{CS}_{2}$, the data are consistent with $35 \%$ of the population achieving an average bent angle of $20 \pm 0.5^{\circ}$. This value is very close to the measurement from Iwasaki, ${ }^{25}$ who determined the average bent angle following strong field excitation of $\mathrm{CS}_{2}$ by angular dependent ionization to be $18 \pm$ $3^{\circ}$ for $\mathrm{CS}_{2}{ }^{3+}$. The angle we have determined $\left(160^{\circ}\right)$ is between the equilibrium angle of the ${ }^{1} B_{2}\left({ }^{1} \Delta_{u}\right)$ and ${ }^{1} A_{2}\left({ }^{1} \Delta_{u}\right)$ states of $\mathrm{CS}_{2}{ }^{60} \mathrm{We}$ have determined the angle and percentage of the population that is deformed by a series of simulations shown in Figure 11. In Figure 11a, we show that as the bend angle increases, the rotational revival shifts toward earlier times (the rotational constant $B$ increases). Because only a portion of the population is bent (the $35 \%$ value arises from the fact that $72 \%$ of the intensity of a Gaussian pulse is contained in the central part of the beam), the rotational revival from all the molecules in the sample needs to be added and then squared to obtain the observed heterodyne detected signal. ${ }^{37,40}$ When we add 35\% of bent molecules, we see changes in the resulting signal, as shown in Figure 11b-f. The simulation in Figure 11e fits our data closely, while the simulations in Figure $11 \mathrm{~d}$ and $\mathrm{f}$ do not. Because the TG method is much more sensitive to neutral molecules than ions, because of their greater polarizability, we can also establish that the molecular deformation takes place in the absence of ionization. We have carried out ab initio 


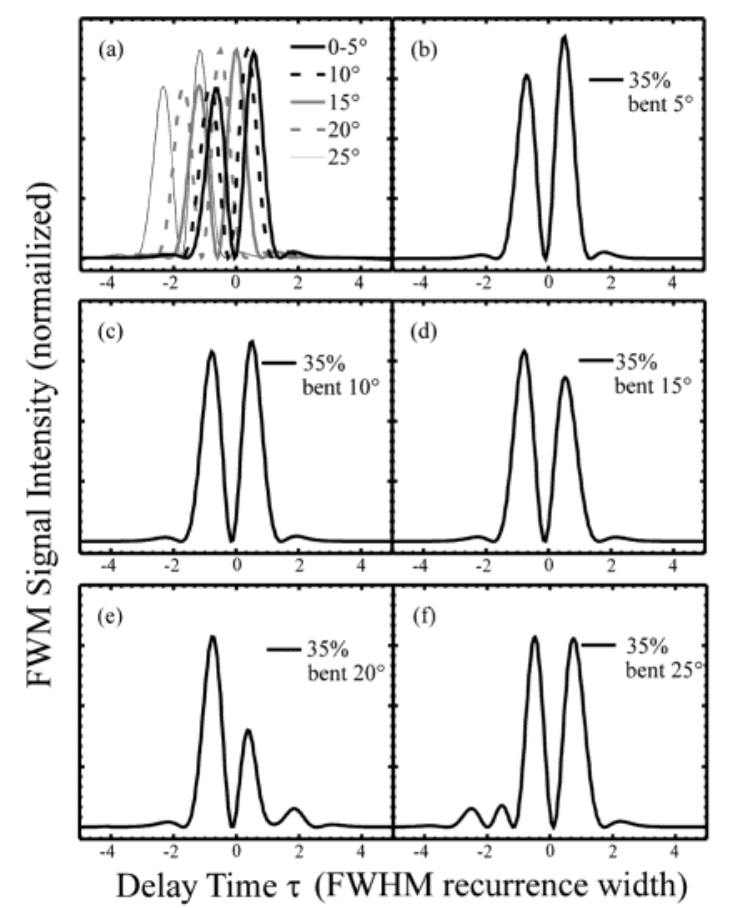

Figure 11. Simulation showing the effects of molecular deformation on the rotational recurrences of a generic molecule. The time axis is in units of the full-width half-maximum of the peaks of the recurrence, with zero being the recurrence time in the linear molecule. Plot a shows recurrences from the bent species, showing the increase in rotational constant as a function of bond angle. Plots $b-f$ show the recurrences of a sample comprised of $35 \%$ bent molecules and $65 \%$ linear molecules for bond angles from $5^{\circ}$ (b) to $25^{\circ}$ (f). The simulation in panel e is in good agreement with our experimental findings shown in Figure 3c.

calculations ${ }^{59}$ to explore molecular structure changes induced by strong $(0.5 \mathrm{~V} / \AA)$ electric fields. We have found that when the field is perpendicular to the molecular axis, a bent molecular structure is preferred. The potential minimum of the polarized molecule no longer corresponds to a linear geometry but to a bent geometry. We have confirmed this by ab initio calculations for $\mathrm{CS}_{2}$ in the presence of a strong $0.5 \mathrm{~V} / \AA$ field. The overall change in the potential energy surface, however, is not large enough to explain the experimental observations.

The bending angle found in the TG data is consistent with the Coulomb explosion data taken in our laboratory with the same laser system. If the molecules remained linear, a case depicted in Figure 12, the signal from the $\mathrm{S}^{+}$ions would show a marked anisotropy. The anisotropy would result from two situations. When the electric field vector is aligned with the TOF axis, the $\mathrm{S}^{+}$ions are ejected toward and away from the detector. Because of the repeller plate, the fragments that are ejected away from the detector are deflected back toward the detector. This leads to a well-known splitting in the ion signal, evident in the zoomed inset of the mass spectrum in Figure 4 for $\mathrm{S}^{2+}$ ions, and used in photofragment spectroscopy analysis. ${ }^{61}$ Note that molecules perpendicular to the field are not affected by the field and yield no ions. When the field is perpendicular to the TOF axis, the $\mathrm{S}^{+}$ions are ejected at a high velocity in a direction that is perpendicular to the detector. Despite the acceleration voltage, the Coulomb repulsion is significant, and in the time it takes those ions to reach the detector plane $(\sim 4 \mu \mathrm{s})$, they are already several centimeters apart and are not detected. For this reason, the $\mathrm{S}^{+}$ions show a distribution that can be simulated by a cosinusoidal function. For linear molecules, the detection of $\mathrm{C}^{+}$ions is not affected by the angle of the polarization vector of the electric field. The predicted

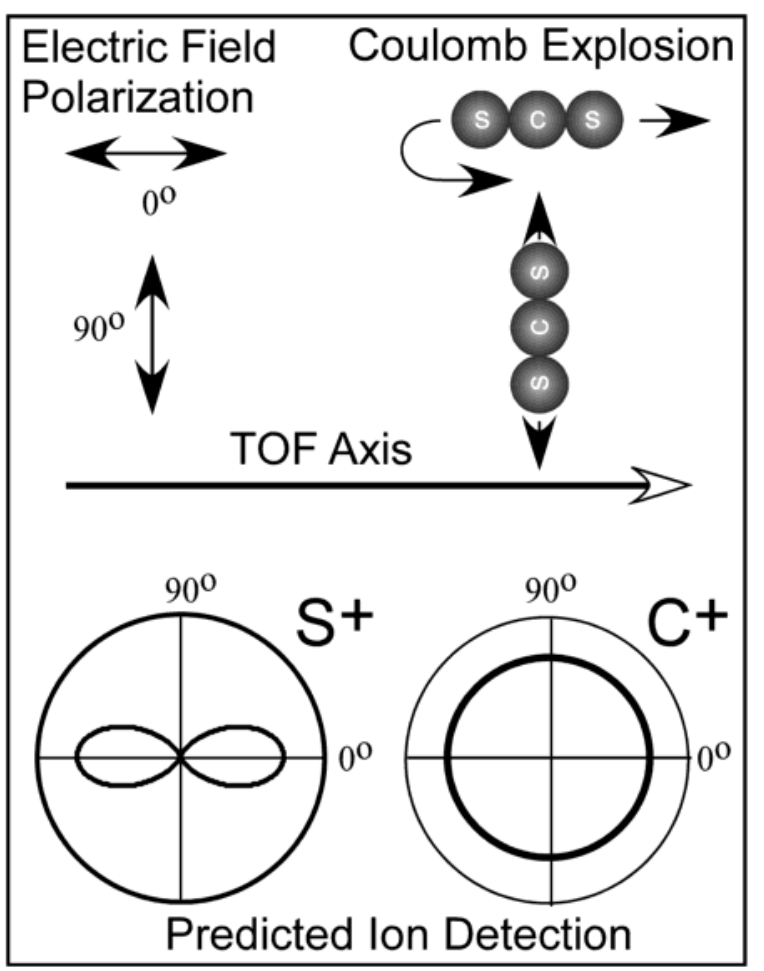

Figure 12. Conceptual drawing of the Coulomb explosion of linear $\mathrm{CS}_{2}$ molecules when the electric field polarization is parallel or perpendicular to the time-of-flight axis. The detection of $\mathrm{S}^{+}$fragment ions is expected to show a marked anisotropy. However, the $\mathrm{C}^{+}$ fragment ion detection is expected to be isotropic.

detection is therefore isotropic, a fact that is not supported by the data shown in the experimental data in Figures 5 and 6.

In Figure 13 we consider the angle dependent detection of fragment ions resulting from bent $\mathrm{CS}_{2}$ molecules. Unless the bending angle is very large, there is almost no change in the anisotropic dependence of the $\mathrm{S}^{+}$ions discussed above for linear molecules; see Figure 13, top. However, for bent molecules the $\mathrm{C}^{+}$signal becomes anisotropic. The anisotropy results from the following observations, based on the assumption that the bending angle is small and that the polarizability of the molecule remains along the two $\mathrm{S}$ atoms. When the electric field vector is aligned parallel to the TOF axis, the bent $\mathrm{CS}_{2}$ molecules can have the two $\mathrm{S}$ atoms along the field or perpendicular to the field. If the molecules are oriented along the field, then the $\mathrm{C}^{+}$ ions are ejected in a direction that is perpendicular to the detector and miss it. When the electric field vector is aligned perpendicular to the TOF axis, the bent $\mathrm{CS}_{2}$ molecules oriented along the field eject $\mathrm{C}^{+}$ions toward the detector and register a maximum signal. When the molecules are oriented perpendicular to the field, the signal is minimal because the perpendicular component of the polarizability is very small for small bend angles. From this analysis so far, it is clear that $\mathrm{C}^{+}$ions are predicted to show anisotropic detection that is perpendicular to that observed for $\mathrm{S}^{+}$ions if the $\mathrm{CS}_{2}$ molecules are bent. The predicted anisotropic ion signal from the Coulomb explosion from bent $\mathrm{CS}_{2}$ molecules is shown in Figure 13. It is difficult to make an accurate determination of the angle from these data without coincidence imaging. A simple simulation of the parallel and perpendicular components of the polarizability as a function of bend angle leads us to estimate the bend angles from the singly and doubly ionized data sets to be $170^{\circ}$ and $155^{\circ}$, respectively. The agreement between the findings of Iwasaki et al. using mass resolved momentum imaging (MRMI) and the $800 \mathrm{~nm}$ excitation wavelength and our angle determination 


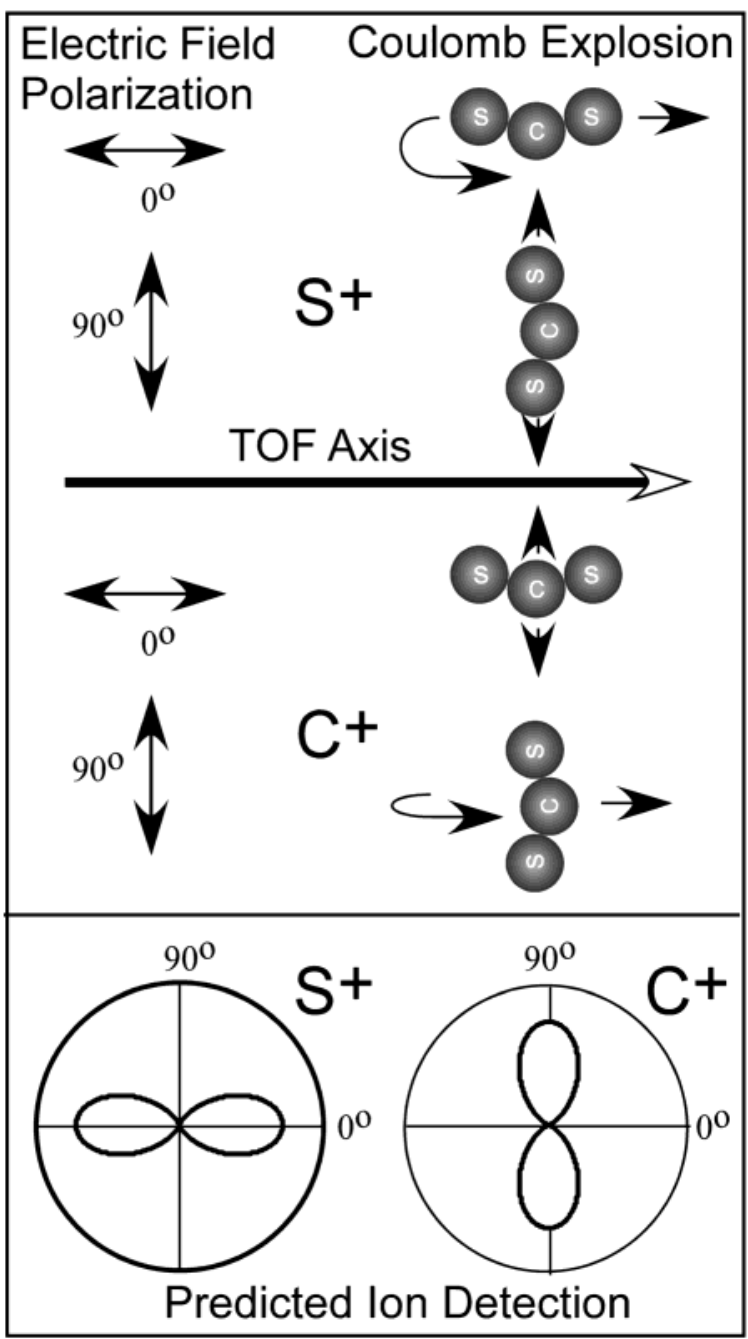

Figure 13. Conceptual drawing of the Coulomb explosion of bent $\mathrm{CS}_{2}$ molecules when the electric field polarization is parallel or perpendicular to the time-of-flight axis. The detection of $\mathrm{S}^{+}$fragment ions is expected to show a marked anisotropy similar to that observed for the linear molecules. However, the $\mathrm{C}^{+}$fragment ion detection is expected to be anisotropic and perpendicular to that observed for the $\mathrm{S}^{+}$fragment ions.

is good. ${ }^{25} \mathrm{We}$ attempted to measure the behavior of $\mathrm{CS}_{2}$ molecules under intense $800 \mathrm{~nm}$ radiation using the TG method in a static cell. Unfortunately, we found that for laser intensities of $10^{12} \mathrm{~W} / \mathrm{cm}^{2}$ and higher a chemical reaction occurred in the cell and all of the sample in the cell decomposed. After a number of attempts, we abandoned this experiment.

The transient grating method is ideal to measure the signal from neutral species with high anisotropic polarizability. For $\mathrm{CO}_{2}$ molecules we found the bond angle of the bent species reached $161^{\circ}$; this value should be compared to the $140^{\circ}$ determined by Hishikawa et al. ${ }^{21}$ As the electric field is increased and ionization takes place, the TG signal decreases and gives way to a background signal caused by the plasma. In Figure $7 \mathrm{e}-\mathrm{g}$, we showed $\mathrm{CO}_{2}$ molecules in the presence of an increasingly strong electric field. Notice that the background increases and that the rotational revivals change shape. Under these conditions, the plasma emits a spectrally shifted light pulse (a spark) that provides a local field to heterodyne the TG signal. ${ }^{52,62}$ Under these conditions, the TG signal changes from being homodyne detected to heterodyne detected. ${ }^{35,36} \mathrm{We}$ find that the weak heterodyne signal corresponds to linear $\mathrm{CO}_{2}$ molecules that are practically unaffected by the electric field. It is very possible that this signal arises from molecules outside

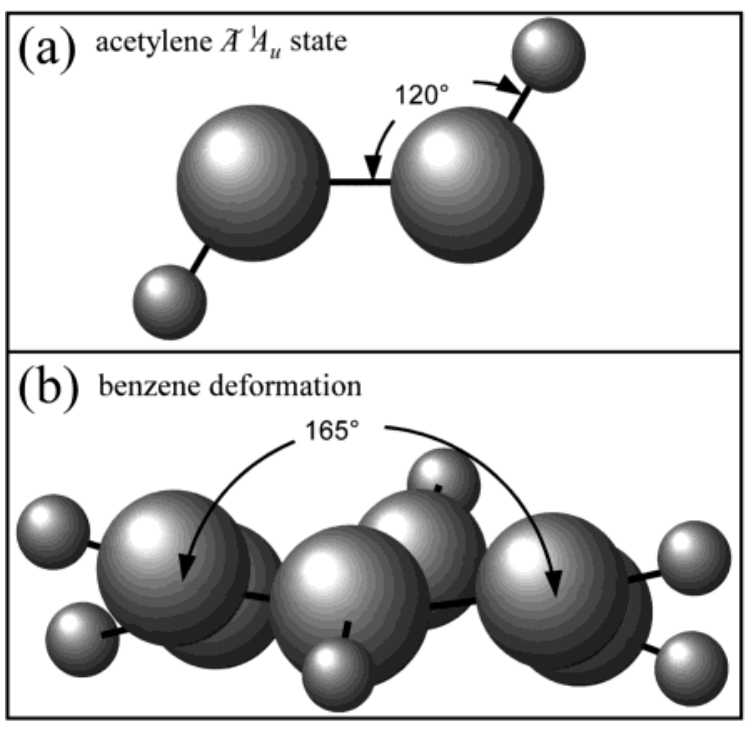

Figure 14. Molecular models depicting the average bent angle observed for acetylene (a) and benzene (b) consistent with the experimental TG data obtained in the presence of strong laser fields.

the main focus of the laser field. Banerjee et al. have explored differences observed in ionization and Coulomb explosion as a function of the electric field intensity profile at the focus of the laser. $^{22}$ Their observation of single ionization in the wings of the focus and highly charged species at the center is consistent with our findings, that is, ionization and plasma formation in the focus with a weak signal from unperturbed molecules from the wings.

Molecular deformation in larger molecules is evident in the experimental data presented here. In the case of acetylene, the electronically excited state responsible for its absorption in the ultraviolet region is known to have a staggered (trans) geometry. ${ }^{63}$ On the basis of this knowledge, we can determine the deviation angle from linearity by fitting of the experimental data. Our data are consistent with bending of $60 \pm 10^{\circ}$, as shown in Figure 14a. Because hydrogen atoms are very light, we are unable to make a more accurate determination of the angle. We have done a similar analysis for benzene in the presence of strong laser fields. The data clearly show a distortion; however, the rotational constant does not change significantly. This implies that the molecule is not stretched in the plane; it is bent out of the plane. The changes are opposite in direction to what would be observed in the presence of a coherent background signal. We attempted to simulate the data by including excited states and ion states of benzene without success. On the basis of the observations, we assume that the molecule assumes a puckered geometry that maintains the molecular symmetry and achieves an angle of $15 \pm 3^{\circ}$ out of planarity; see Figure 14b. The symmetry of the motion is imposed by the lack of asymmetry rotational recurrences in the transient. Shimizu et al. studied the molecular deformations of benzene under strong field excitation by Coulomb explosion; their molecular dynamics simulations predicted out of plane motion of the carbon atoms. ${ }^{64}$ This Raman active out of plane motion with $b_{2 g}$ symmetry couples with some of benzene's forbidden transitions to excited states $\left({ }^{1} \mathrm{~B}_{2 \mathrm{u}},{ }^{1} \mathrm{~B}_{1 \mathrm{u}},{ }^{1} \mathrm{E}_{1 \mathrm{u}}\right.$, and $\left.{ }^{1} \mathrm{~B}_{1 \mathrm{~g}}\right)$, all of which have lowered barriers for out of plane motion. ${ }^{65-67}$

\section{Conclusions}

In this paper we have presented a study about the behavior of polyatomic molecules in the presence of strong off-resonance 
ultrashort laser fields. Our experimental data are consistent with alignment of the molecules. This alignment results from a "kick" induced by the electric field acting on the polarizability of the molecule. Our data provide clear evidence of significant molecular structure deformation resulting from the interaction with the strong field. These observations are consistent with Coulomb explosion measurements from our group and from other groups. ${ }^{21,25,64}$

Two explanations for this deformation were discussed, one involving excited electronic states with bent geometry, and one dealing strictly with perturbations to the ground state potential. We plan to explore evidence of electronic state involvement by laser induced fluorescence after strong electric field interaction. If the TG measurements are reflecting a large population of excited state molecules, fluorescence from these states should be experimentally observable. Probing for the presence of ground state molecules with significant vibrational excitation will be more difficult because the bending motion is not a Raman active mode. We plan to run more electronic structure calculations with the Piecuch group to explore deformations of the excited states in the presence of strong fields. These efforts will be extended to other molecular systems.

Recent experiments on laser control of photodissociation reactions using shaped pulses require a careful evaluation of the effect of strong off-resonance laser fields on the electronic potentials of molecules. ${ }^{68,69}$ In particular, it is important to determine to what extent these fields align and deform the neutral species. In particular, it would be important to determine if the observed chemistry takes place in the ground or electronic excited states of neutral molecules or if the chemistry takes place after ionization. The study presented here, especially the TG measurements on the neutral molecules, indicates that the electric field can have a major influence on the molecular structure without causing ionization. Our study and that of Banarjee et al. highlight differences caused by the gradient in the electric field intensity of Gaussian pulses, an aspect that has not been addressed in strong field laser control. The temporal envelope of the excitation pulse has not received sufficient attention in studies of molecular deformation. In our case the pulse duration ( $60 \mathrm{fs}$ ) is similar to the bending period of ground state $\mathrm{CS}_{2}$. Therefore, during the laser pulse the nuclei have time to move away from linear geometry. Once the field is gone, the ground state potential is restored and the molecule is left vibrationally excited. It would be interesting to explore the effects of pulse duration and excitation with different temporal envelopes. This research could yield methodology to figure out the design of the field to carry out a specific chemical reaction such as isomerization.

In conclusion, the time resolved TG method is ideal to measure molecular alignment and molecular structure deformation induced by strong laser fields. The method provides a high degree of accuracy, and it has been used to determine structural deformation in $\mathrm{CS}_{2}, \mathrm{CO}_{2}$, acetylene, and benzene. It is important to point out that the TG measurement is made by the time delayed probe pulse, which has minimal influence on the molecular system and arrives a long time after the strong field has been turned off. This is in contrast to other ionization-based experiments, where the strong field affects the molecule and releases the charged particles. The TG measurements here are consistent with alignment and bending dynamics that take place in neutral molecules, in the absence of ionization. On the basis of the findings presented here, it will be interesting to explore in more detail the deformation of larger polyatomic molecules.
Acknowledgment. This work was supported by National Science Foundation Grant CHE-0135581. We are especially grateful for the calculations of the potential energy surfaces of $\mathrm{CS}_{2}$ in the presence of an electric field carried out by Professor Piotr Piecuch. We are also grateful for stimulating discussions on the influence of strong laser fields on molecules with Tamar Seideman and Vadim V. Lozovoy. M.D. is a Camille Dreyfus Teacher-Scholar.

\section{References and Notes}

(1) Friedrich, B.; Herschbach, D. Phys. Rev. Lett. 1995, 74.

(2) Kim, W.; Felker, P. M. J. Chem. Phys. 1996, 104, 1147.

(3) Dion, C. M.; Keller, A.; Atabek, O.; Bandrauk, A. D. Phys. Rev. A 1999, 59, 1382

(4) Yamanouchi, K. Science 2002, 295, 1659

(5) Friedrich, B. Phys. Rev. A 2000, 61, 025403.

(6) Friedrich, B.; Herschbach, D. J. Phys. Chem. 1995, 99, 15686. L497.

(7) Normand, D.; Lompre, L. A.; Cornaggia, C. J. Phys. B 1992, 25 ,

(8) Dietrich, P.; Strickland, D.; Laberge, M.; Corkum, P. B. Phys. Rev. A 1993, 47, 2305.

(9) Kim, W. S.; Felker, P. M. J. Chem. Phys. 1997, 107, 2193

(10) Sakai, H.; Safvan, C. P.; Larsen, J. J.; Hilligsøe, K. M.; Hald, K.; Stapelfeldt, H. J. Chem. Phys. 1999, 110, 10235.

(11) Larsen, J. J.; Wendt-Larsen, I.; Stapelfeldt, H. Phys. Rev. Lett. 1999, 1123.

(12) Posthumus, J. H.; Plumridge, J.; Thomas, M. K.; Codling, K.; Frasinski, L. J.; Langley, A. J.; Taday, P. F. J. Phys. B: At., Mol. Opt. Phys. 1998, 31, L553.

(13) Villeneuve, D. M.; Aseyev, S. A.; Dietrich, P.; Spanner, M.; Corkum, P. B. Phys. Rev. Lett. 2000, 85, 545.

(14) Seideman, T. J. Chem. Phys. 1995, 103, 7887.

(15) Brown, E. J.; Pastirk, I.; Dantus, M. J. Phys. Chem. A 2001, 105, 8004

(16) Ortigoso, J.; Rodrigues, M.; Gupta, M.; Friedrich, B. J. Chem. Phys. 1999, 110,3870 .

(17) Cornaggia, C.; Salin, F.; Le Blanc, C. J. Phys. B: At., Mol. Opt. Phys. 1996, 32, 5557.

(18) Cornaggia, C. Phys. Rev. A 1996, 54, R2555.

(19) Hishikawa, A.; Iwamae, A.; Hoshina, K.; Kono, M.; Yamanouchi, K. Chem. Phys. Lett. 1998, 282, 283.

(20) Hishikawa, A.; Iwamae, A.; Hoshine, K.; Kono, M.; Yamanouchi, K. Chem. Phys. 1998, 231, 315.

(21) Hishikawa, A.; Iwamae, A.; Yamanouchi, K. Phys. Rev. Lett. 1999 $83,1127-1130$

(22) Banerjee, S.; Ravindra Kumar, G.; Mathur, D. J. Phys. B: At., Mol. Opt. Phys. 1999, 32, 4277.

(23) Banerjee, S.; Ravindza Kumar, G.; Mathur, D. Phys. Rev. A 1999, 60, R3369.

(24) Iwamae, A.; Hishikawa, A.; Yamanouchi, K. J. Phys. B: At., Mol. Opt. Phys. 2000, 33, 223

(25) Iwasaki, A.; Hishikawa, A.; Yamanouchi, K. Chem. Phys. Lett. 2001, 346, 379 .

(26) Graham, P.; Ledingham, K. W. D.; Singhal, R. P.; McCanny, T.; Hankin, S. M.; Fang, X.; Smith, D. J.; Kosmidis, C.; Tzallas, P.; Langley, A. J.; Taday, P. F. J. Phys. B: At., Mol. Opt. Phys. 1999, 32, 5557.

(27) Couris, S.; Koudoumas, E.; Leach, S.; Fotakis, C. J. Phys. B: At., Mol. Opt. Phys. 1999, 32, L439.

(28) Frasinski, J. J.; Codling, K.; Hatherly, P. A. Phys. Lett. A 1989 , $142,499$.

(29) Hishikawa, A.; Iwamae, A.; Yamanouchi, K. J. Chem. Phys. 1999 111,8871

(30) Hasegawa, H.; Hishikawa, A.; Yamanouchi, K. Chem. Phys. Lett. 2001, 349, 57.

(31) Sanderson, J. H.; El-Zein, A.; Bryan, W. A.; Newell, W. R.; Langley, A. J.; Taday, P. F. Phys. Rev. A 1999, 59, R2567.

(32) Bryan, W. A.; Sanderson, J. H.; El-Zein, A.; Newell, W. R.; Taday,

P. F.; Langley, A. J. J. Phys. B: At., Mol. Opt. Phys. 2000, 33, 745.

(33) Wu, C.; Ren, H.; Liu, T.; Ma, R.; Yang, H.; Jiang, H.; Gong, Q. Int. J. Mass Spectrom. 2002, 216, 249.

(34) Heritage, J. P.; Gustafson, T. K.; Lin, C. H. Phys. Rev. Lett. 1975, 34, 1299.

(35) Morgen, M.; Price, W.; Ludowise, P.; Chen, Y. J. Chem. Phys. 1995, 102, 8780-8789.

(36) Morgen, M.; Price, W.; Hunziker, L.; Ludowise, P.; Blackwell, M.; Chen, Y. Chem. Phys. Lett. 1993, 209, 1-9.

(37) Brown, E. J.; Zhang, Q. G.; Dantus, M. J. Chem. Phys. 1999, 110, $5772-5788$

(38) Dantus, M. Faraday Discuss. 1999, 113, 471 
(39) Comstock, M.; Pastirk, I.; Dantus, M. Ultrafast transient-grating study of molecules after high-intensity excitation; Ultrafast Phenomena XII; Springer Verlag: Berlin, 2000.

(40) Dantus, M. Аnnu. Rev. Phys. Chem. 2001, 52, 639.

(41) Joireman, P. W.; Ohline, S. M.; Felker, P. M. J. Phys. Chem. A 1998, 102, 4481-4494.

(42) Frey, H. M.; Beaud, P.; Gerber, T.; Mischler, B.; Radi, P. P.; Tzannis, A. P. J. Raman Spectrosc. 2000, 31, 71-76.

(43) Jarzeba, W.; Matylitsky, V. V.; Weichert, A.; Riehn, C. Phys. Chem. Chem. Phys. 2002, 4, 451-454.

(44) Lang, T.; Motzkus, M.; Frey, H. M.; Beaud, P. J. Chem. Phys. 2001, 115, 5418-5426.

(45) Seideman, T. Phys. Rev. Lett. 1999, 83, 4971-4974.

(46) Mukamel, S. Principles of Nonlinear Optical Spectroscopy; Oxford University Press: New York, 1995.

(47) Equation 1 also holds true for the $\Delta K=0$ Raman transition of a symmetric top molecule, and it can be applied to nearly symmetric top molecules provided the effective $B$ constant $B_{\text {eff }}=(B+C) / 2$ is used. Herzberg, G. Molecular Spectra and Structure: Volume III Electronic Spectra and Electronic Structure of Polyatomic Molecules; Krieger Publishing Company: Malabar, FL, 1991; pp 106-108.

(48) For electronic states of gerade symmetry, Bosonic nuclei have $g_{J}=(2 I+1)(I+1)$ if $J$ is even and $g_{J}=(2 I+1) I$ if $J$ is odd. Fermionic nuclei in gerade electronic states have $g_{J}=(2 I+1) I$ if $J$ is even and $g_{J}=$ $(2 I+1)(I+1)$ if $J$ is odd. For ungerade electronic states, the ratio of odd to even states is reversed. Here $I$ is the nuclear spin of indistinguishable particles exchanged during rotation. For heteronuclear diatomics, interchange symmetry does not apply and all states are equally weighted. For more information see: Townes, C. H.; Schawlow, A. L. Microwave Spectroscopy; Dover Publications: New York, 1975; pp 102-105.

(49) Rosca-Pruna, F.; Vrakking, M. J. J. J. Chem. Phys. 2002, 116, $6579-6588$

(50) Rosca-Pruna, F.; Vrakking, M. J. J. J. Chem. Phys. 2002, 116, $6567-6578$

(51) Lozovoy, V. V.; Comstock, M.; Dantus, M. Four-wave mixing and coherent control. In Laser Control and Manipulation of Molecules; Bandrauk, A. D., Fujimura, Y., Gordon, R. J., Eds.; American Chemical Society: Washington DC, 2002; Vol. 821, pp 61-80.
(52) Pastirk, I.; Comstock, M.; Walowicz, K. A.; Lozovoy, V. V.; Dantus, M. 2D (time-frequency) femtosecond four-wave mixing at $10^{14}$ W/cm²: Molecular and electronic response; Symposium on Optical Pulse and Beam Propagation III; Photonics West: 2001

(53) Matylitsky, V. V.; Jarzeba, W.; Riehn, C.; Brutschy, B. J. Raman Spectrosc. 2002, 33, 877.

(54) Giusti-Suzor, A.; Mies, F. H.; DiMauro, L. F.; Charron, E.; Yang, B. J. Phys. B 1995, 28, 309 .

(55) Bandrauk, A. Molecules in Intense Laser Fields; M. Dekker Publishing: New York, 1993.

(56) Tseng, D. C.; Posthusta, R. D. J. Chem. Phys. 1994, 100, 7481.

(57) Zhang, Q. C.; Vaccaro, P. H. J. Phys. Chem. 1995, 99, 1799.

(58) Brown, S. T.; Van Huis, T. J.; Hoffman, B. C.; Schaeffer, H. F., III. Mol. Phys. 1999, 96, 693.

(59) Calculation performed using the $\operatorname{CCSD}(\mathrm{T})$ method with the augcc-PVDZ basis set. The lowest two orbitals (correlating with the 1s orbitals in $\mathrm{C}$ and $\mathrm{S}$ ) were frozen in the calculations. We also calculated the states in the presence of a $0.5 \mathrm{~V} / \AA$ field parallel or perpendicular to the molecular axis.

(60) Brasen, G.; Leidicker, M.; Demtroder, W.; Shimamoto, T.; Kato, H. J. Chem. Phys. 1998, 109, 2779.

(61) Sander, R. K.; Wilson, K. R. J. Chem. Phys. 1975, 63, 4242.

(62) Grimberg, I.; Lozovoy, V. V.; Dantus, M.; Mukamel, S. J. Phys. Chem. A 2002, 106, 697-718.

(63) Herzberg, G. Molecular Spectra and Molecular Structure Vol. III Electronic Spectra and Electronic Structure of Polyatomic Molecules; Krieger Publishing Company: Malabar, FL, 1991.

(64) Shimizu, S.; Zhakhovski, V.; Fuminobu, S.; Okihara, S.; Sakabe, S.; Nishihara, K.; Izawa, Y.; Yatsuhashi, T.; Nakashima, N. J. Chem. Phys. 2002, 117, 3180 .

(65) Christiansen, O.; Koch, H.; Halkier, A.; Jorgenson, P.; Helgaker, T.; Sanchez de Meras, A. J. Chem. Phys. 1996, 105, 6921.

(66) Borges, I., Jr.; Varandas, A. J. C.; Rocha, A. B.; Bielschowsky, C. E. THEOCHEM 2003, 621, 99.

(67) Norman, P.; Agren, H. THEOCHEM 1997, 401, 107.

(68) Levis, R. J.; Menkir, G. M.; Rabitz, H. Science 2001, 292, 709.

(69) Assion, A.; Baumert, T.; Bergt, M.; Brixner, T.; Kiefer, B.; Seyfried, V.; Srohle, M.; Gerber, G. Science 1998, 282, 919. 